\title{
Scattering from Chirally Coated Oblate Spheroids - A Study in RCS Modifications
}

\author{
R. Sharma and N. Balakrishnan \\ Department of Aerospace Engineering, Indian Institute of Science \\ Bangalore 560 012, INDIA
}

\section{ABSTRACT}

In this paper extended boundary condition method has been formulated to compute the scattering from three dimensional dielectric scatterers coated by a chiral material. The applicability of this method to oblate spheroidal shape is also discussed.

\section{INTRODUCTION}

The lack of geometric symmetry between an object and its mirror image is referred to as chirality, and the mirror image of such a chiral object cannot be made to coincide with the object itself by any operation involving rotations and/or translations. In recent times a lot of attention has been focussed towards evaluation and reduction of radar cross section (RCS) using chiral materials. Bohren [1] studied the scattering from a chiral sphere for the first time, using the constitutive equations appropriate for an optically active isotropic medium. The scattering and absorption from chiral non-spherical objects was studied by Lakhtakia et.al [2]. Uslenghi [3] evaluated the scaterring from the chirally coated sphere. Scattering from chirally coated planar surfaces with an aim to reduce the RCS, was analysed by Jaggard [4].

The present work is directed towards the study of electromagnetic scattering from three dimensional non-spherical chirally coated scatterers. The analysis is carried out using extended boundary condition method (EBCM). This method was first developed by Waterman [5] using the Huygen's - Poincare method to evaluate the scaterring from homogeneous nonspherical bodies. Barber and Yeh [6] modified the analysis using the schelkunoff's field equivalence principle, and their formulation is convenient to program on a computer and is applicable to dielectric bodies. Barber and Wang [7] applied this method to three dimensional electromagnetic scattering problems involving multi-layered dielectric objects. In this paper, Barber and Wang's [7] formulation has been modified to take into account the chiral nature of the outer layer. The modified formulation is used to evaluate the scattered fields and the radar cross section of chirally coated non-spherical dielectric bodies.

\section{FORMULATION OF THE PROBLEM}

The constitutive relations in a chiral medium get modified as [8]

$$
\begin{aligned}
& \bar{D}=\epsilon \bar{E}+\beta \epsilon \nabla \times \bar{E} \\
& \bar{B}=\mu \bar{H}+\beta \mu \nabla \times \overline{\mathrm{H}}
\end{aligned}
$$

where $\epsilon, \mu$ and $\beta$ are the permittivity, permeability and chirality parameter of the medium.

Following Bohren [1] the EM fields are transformed to

$$
\left[\begin{array}{l}
\overline{\mathrm{E}} \\
\overline{\mathrm{H}}
\end{array}\right]=[\mathrm{A}]\left[\begin{array}{l}
\overline{\mathrm{Q}}_{\mathrm{L}} \\
\overline{\mathrm{Q}}_{\mathrm{R}}
\end{array}\right]
$$


where $\overline{\mathbf{Q}}_{\mathbf{L}}$ and $\overline{\mathbf{Q}}_{\mathrm{R}}$ are the LCP and the RCP fields and satisfy the conditions

$$
\begin{aligned}
& \left(\nabla^{2}+\mathrm{k}_{\mathrm{L}}{ }^{2}\right) \overline{\mathrm{Q}}_{\mathrm{L}}=0 \\
& \left(\nabla^{2}+\mathrm{k}_{\mathrm{R}}{ }^{2}\right) \overline{\mathrm{Q}}_{\mathrm{R}}=0
\end{aligned}
$$

along with the auxiliary conditions

$\nabla \times \bar{Q}_{L}=k_{L} \bar{Q}_{L} ; \nabla \cdot \bar{Q}_{L}=0$
$v \times \bar{Q}_{R}=k_{R} \bar{Q}_{R} ; \nabla \cdot \bar{Q}_{R}=0$

In equation

(2) the matrix

A is given as

$[A]=\left[\begin{array}{cc}1 & a_{R} \\ a_{L} & 1\end{array}\right]$

where $k_{L}=k /(1-k \beta)$;

$a^{L}=-j \sqrt{L} / \mu ;$

$\dot{k}_{\mathrm{R}}=k /(1+k \beta)$
$\mathrm{a}_{\mathrm{R}}=-j /(\sqrt{ } \boldsymbol{\omega} / \mu)$$;$

Thus, the electromagnetic fields existing in the chiral medium are given by

$\overline{\mathrm{E}}=\overline{\mathrm{Q}}_{\mathrm{L}}+\mathrm{a}_{\mathrm{R}} \overline{\mathrm{Q}}_{\mathrm{R}} ;$
$\overline{\mathrm{H}}=\overline{\mathrm{Q}}_{\mathrm{R}}+\mathrm{a}_{\mathrm{L}} \overline{\mathrm{Q}}_{\mathrm{L}}$

Consider a chirally coated body, say an oblate spheroid as shown in Fig. 1.

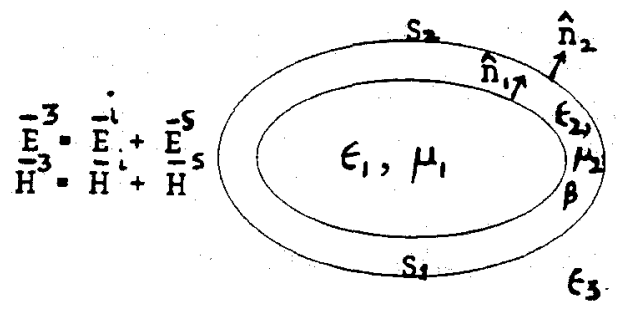

$\bar{J}_{i}, \bar{M}_{i}$

Fig.l Chirally coated oblate spheroid

It can be treated as a two layered object which is characterised by the constitutive parameters $\boldsymbol{\epsilon}_{1}, \boldsymbol{\mu}_{1}$ for the inner layer and $\boldsymbol{\epsilon}_{2}, \mu_{2}$ and $\beta$ for the chiral layer. The permittivity of the surrounding medium is $\varepsilon_{3}$ which maybe free space. For the sake of simplicity the permeability is assumed to be uniform throughout the entire space and equal to $\mu_{0}$ the permeability of the free space. Applying the field equivalence principle results in the reduction of the two layered scattering problem into three sub problems as shown in Fig. 2.

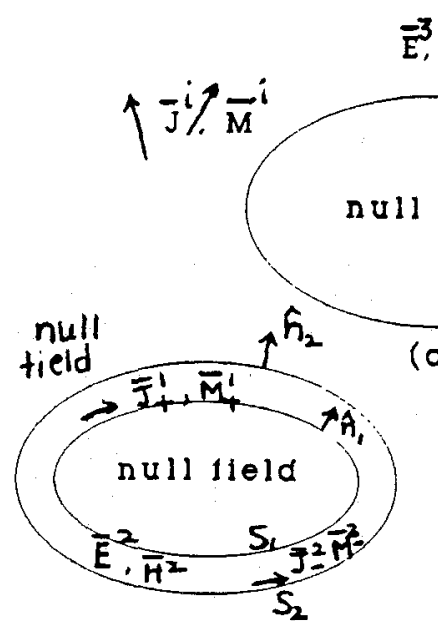

(b)
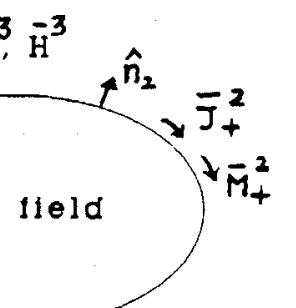

Fig.2 Application 01 equivalence principle; Original scattering problem is replaced by three sub-problems

Modifying the Barber and Hang's [7] formulation for the chiralIy coated body (Fig.1) we have the following integral equations;

$\overline{\mathrm{E}}^{3}(\overline{\mathrm{R}})=\overline{\mathrm{E}}^{i}(\bar{R})+\mathrm{V} \times \int_{S_{2}}\left(\hat{\mathrm{n}}_{2} \mathrm{X} \overline{\mathrm{E}}_{+}^{3}\right) \cdot$ $\overline{\bar{G}}\left(k_{3} \bar{R}\right) d s^{\prime}-V \times V X\left(1 / j w \epsilon_{3}\right) \int_{S_{2}}\left(\hat{n}_{2}\right.$ $\left.X \bar{H}_{+}^{3}\right) \cdot \overline{\bar{G}}\left(k_{3} \bar{R}\right) d s^{\prime} \quad$ outside $s_{2}$ $=0$ inside $S_{Z_{5}}$

$\overline{\mathrm{E}}^{2}(\overline{\mathrm{R}})=\mathrm{V} \times \int_{\mathrm{S}_{2}}\left(-\hat{\mathrm{n}}_{2} \times \overline{\mathrm{E}}_{-}^{2}\right) \cdot \overline{\bar{G}}\left(\mathrm{k}_{2} \overline{\mathrm{R}}\right) \mathrm{d} \mathbf{s}^{\prime}$ - $\nabla \times \nabla \times \int_{S_{2}}\left(1 / j w \epsilon_{2}\right)\left(-\hat{n}_{2} \times \bar{H}_{-}^{2}\right)$. $\overline{\bar{G}}\left(k_{2} \bar{R}\right) d s^{\prime}+\nabla \times \int_{s_{1}}\left(\hat{n}_{1} \times \bar{E}_{+}^{2}\right) \cdot$ $\overline{\bar{G}}\left(k_{2} \bar{R}\right) d s^{\prime}-\nabla X \nabla X \int_{s_{1}}\left(1 / j w \varepsilon_{2}\right)\left(\hat{n}_{1} \times \bar{H}_{+}^{2}\right) \cdot$ $\overline{\bar{G}}\left(k_{2} \bar{R}\right) d s^{\prime}$ between $s_{1}$ and $s_{2}$ $=0 \quad$ inside $s_{1}$ $\overline{\mathbf{G}}\left(\mathbf{k}_{\mathbf{i}} \overline{\mathbf{R}}\right)$ is the Green's dyadic 
for that medium. $\bar{R}=\left|r-r^{\prime}\right|$ and $\bar{r}$ and $\bar{r}$ ' are position vectors from an interior origin to field and source points, respectively. The ' +1 and $1-1$ subscripts indicate external and internal fields respectively, evaluated at the surface. The superscripts in $E$ and $H$ denote the fields in the regions as shown in Fig.1. An equation for $E^{1}(R)$ could also be written but is redundant. Enforcing the boundary conditions that the tangential electric and magnetic fields are continuous across the surfaces $S_{1}$ and $S_{2}$ in (5) and (6), gives a set of integral equations,

$\bar{E}^{3}(\bar{R})=\bar{E}^{i}+v \times \int_{S_{2}}\left(\hat{n}_{2} \times \bar{E}_{-}^{2}\right) \cdot$

$\overline{\bar{G}}\left(k_{3} \bar{R}\right) d s^{\prime}-\nabla \times x^{S_{2}} \quad x_{S_{2}}\left(1 / j w \epsilon_{3}\right)$ $\left(\hat{n}_{2} \times \bar{H}_{-}^{2}\right) \cdot \overline{\bar{G}}\left(k_{3} \bar{R}\right)$ ds' outside $s_{2}$

$=0$

inside $s_{2}$

$\overline{\mathrm{E}}^{2}(\bar{R})=\mathrm{V} \mathrm{X}_{S_{2}}\left(-\hat{\mathrm{n}}_{2} \times \overline{\mathrm{E}}_{-}^{2}\right)$

$\overline{\bar{G}}\left(k_{2} \bar{R}\right) d s^{\prime}-v \times \nabla \times \int_{2}\left(1 / j w \epsilon_{2}\right)$

$\left(\hat{n}_{2} \times \bar{H}_{-}^{2}\right) \cdot \overline{\bar{G}}\left(k_{2} \bar{R}\right) d s^{\prime}+V X \int_{s_{1}}\left(\hat{n}_{1}\right.$

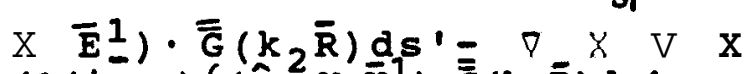

$\left(1 / j w \epsilon_{2}\right) \int_{s_{1}}\left(\hat{n}_{1} x \bar{H}_{-}\right) \cdot \overline{\bar{G}}\left(k_{2} \bar{R}\right) d s^{\prime}$

$=0 \quad$ outside $S_{2}$ and

inside $S_{1}$

To solve the integral equations, the fields are expanded in terms of the vector spherical harmonics $M$ and $N$ [9]

$\bar{E}^{i}(r)=\Sigma D_{\mu}\left[a_{\mu} \bar{M}_{\mu}^{1}\left(k_{3} r\right)+b_{\mu}\right.$

$\left.\overline{\mathrm{N}}_{\mu}{ }^{1}\left(\mathrm{k}_{3} \mathrm{r}\right)\right]$

$\overline{\mathrm{E}}^{\mathrm{s}}(r)=\Sigma D_{\mu}\left[\alpha_{\mu} \overline{\mathrm{M}}_{\mu}^{3}\left(\mathrm{k}_{3} r\right)+B_{\mu}\right.$

$\left.\overline{\mathrm{N}}_{\mu}^{3}\left(\mathrm{k}_{3} \mathrm{r}\right)\right]$

$\overline{\mathrm{E}}^{1}(r)=\Sigma \mathrm{D}_{\mu}\left[\mathrm{C}_{\mu} \overline{\mathrm{M}}_{\mu}^{1}\left(\mathrm{k}_{3} r\right)+\mathrm{d}_{\mu}\right.$

$\left.\overline{\mathrm{N}}_{\mu}{ }^{1}\left(\mathrm{k}_{3} r\right)\right]$

(9c) where $\Sigma=\sum_{\substack{\sigma=\text { even } \\ \text { or odd }}} \stackrel{n}{m=0} \sum_{n=1}^{\infty}$

D is the normalization constant. The free space dyadic Green's function is expanded as in Morse and Feshbach [10]. The superscripts 1 and 3 indicate Bessel and Hankel function radial dependence respectively. The index $n$ is truncated at some value $N$ depending on the accuracy requirements. To evaluate the fields in the chiral layer $\bar{Q}_{L}$ and $\bar{Q}_{R}$ are expanded in terms of vector spherical harmonics [11]

$\overline{\mathrm{Q}}_{\mathrm{L}}=\Sigma \mathrm{D}_{\mu}\left[\mathrm{f}_{\mu},\left[\overline{\mathrm{M}}_{\mu},{ }^{1}\left(k_{\mathrm{L}} \mathrm{r}\right)+\right.\right.$

$\left.\overline{\mathrm{N}}_{\mu},{ }^{1}\left(k_{L} r\right)\right]+\mathrm{p}_{\mu},\left[\bar{M}_{\mu},{ }^{3}\left(k_{L} r\right)+\right.$

$\left.\left.\overline{\mathrm{N}}_{\mu},{ }^{3}\left(k_{L} r\right)\right]\right]$

$\overline{\mathrm{Q}}_{\mathrm{R}}=\Sigma D_{\mu} \cdot\left[g_{\mu},\left[\overline{\mathrm{M}}_{\mu}, 3\left(\mathrm{k}_{\mathrm{R}} \mathrm{r}\right)\right.\right.$ -

$\left.\overline{\mathrm{N}}_{\mu},{ }^{3}\left(\mathrm{k}_{\mathrm{R}} \mathrm{r}\right)\right]+\mathrm{q}_{\mu},\left[\overline{\mathrm{M}}_{\mu},{ }^{3}\left(\mathrm{k}_{\mathrm{R}} \mathrm{r}\right)-\right.$

$\left.\left.\bar{N}_{\mu} \cdot 3\left(k_{R} r\right)\right]\right]$

Using equation (10) along with equation (4) the fields in region (2) can be evaluated.

The dyadic Green's function in chiral region is given as in [11]

$\bar{G}\left(k_{2} \bar{R}\right)=\bar{\Gamma}_{L}+\bar{\Gamma}_{R}$ where

$\bar{\Gamma}_{L}=\left(j k_{L} / \pi\right) \Sigma D_{\mu}\left[\bar{M}_{\mu}, 3\left(k_{L} r\right)+\right.$

$\left.\overline{\mathrm{N}}_{\mu},{ }^{3}\left(k_{L} r\right)\right]\left[\overline{\mathrm{M}}_{\mu},{ }^{1}\left(k_{L} r\right)+\bar{N}_{\mu},{ }^{1}\left(k_{L} r\right)\right]$

and

$\bar{\Gamma}_{R}=\left(j k_{R} / \pi\right) \Sigma D_{\mu}\left[\bar{M}_{\mu}, 3\left(k_{R} r\right)-\right.$

$\left.\overline{\mathrm{N}}_{\mu},^{3}\left(k_{\mathrm{R}} r\right)\right]\left[\overline{\mathrm{M}}_{\mu},{ }^{1}\left(k_{\mathrm{R}} r\right)-\overline{\mathrm{N}}_{\mu},{ }^{1}\left(k_{\mathrm{R}} r\right)\right]$

Inside the surface $s_{2}$, the total field is zero as in equation (7). Substituting (10), (11) and (12) in equations (7) and (8)

$-\bar{E}^{I}(\bar{R})=\nabla \times \int_{S_{2}}\left(\hat{n}_{2} \times \bar{E}_{-}^{2}\right) \cdot \overline{\bar{G}}\left(k_{3} \bar{R}\right) d s^{\prime}$

$-\nabla \times \nabla \times \int_{S_{2}}\left(1 / j w \epsilon_{3}\right) \cdot\left(\hat{n}_{2} \times \bar{H}^{2}\right)$.

$\overline{\bar{G}}\left(\mathrm{k}_{3} \overline{\mathrm{R}}\right) \mathrm{ds} \mathrm{s}^{\prime}$ 
Writing it in matrix form

$$
\begin{aligned}
& {\left[\begin{array}{l}
a \\
b
\end{array}\right]=\left[\begin{array}{ll}
Q_{11 a} & Q_{12 a} \\
Q_{21 a} & Q_{22 a}
\end{array}\right] \cdot\left[\begin{array}{l}
f \\
g
\end{array}\right]} \\
& +\left[\begin{array}{ll}
Q_{11 a^{\prime}} & Q_{12 a^{\prime}} \\
Q_{21 a^{\prime}} & Q_{22 a^{\prime}}
\end{array}\right] \cdot\left[\begin{array}{l}
p \\
q
\end{array}\right] \\
& Q_{11 a}=\left(-j k_{3} 2 / \pi\right) \int_{S_{2}} \bar{N}_{\mu}{ }^{3}\left(k_{3} r\right) \text {. } \\
& {\left[\hat{n}_{2} \times \bar{M}_{\mu}{ }^{1}\left(k_{L} r\right)+\hat{n}_{2} \times \bar{N}_{\mu}{ }^{1}\left(k_{L} r\right)\right]} \\
& +a_{L} j v \mu_{0} / \epsilon_{0} \bar{M}_{\mu},{ }^{3}\left(k_{3} r\right)\left[\hat{n}_{2} X\right. \\
& \left.\bar{M}_{1 \mu}{ }^{I}\left(k_{L} r\right)+\hat{n}_{2} \times \bar{N}_{\mu}{ }^{I}\left(k_{L} r\right)\right\} d s^{\prime}
\end{aligned}
$$

Similar expressions can be written for other elements of Q' matrices. The scattered field is given as

$$
\begin{aligned}
& \bar{E} s(\bar{R})=\nabla \times \int\left(\hat{n}_{2} \times \bar{E}_{-}^{2}\right) \overline{\bar{G}}\left(k_{3} \bar{R}\right)- \\
& V \times V \times \int_{s_{2}}^{S}\left(1 / j w \epsilon_{3}\right)\left(\hat{n}_{2} \times \bar{H}_{-}^{2}\right) \cdot \\
& \bar{G}\left(k_{3} \bar{R}\right) d s^{\prime}
\end{aligned}
$$$$
\text { and can be evaluated once the }
$$
solution to eqn. (15) is obtained. The RCS then can be obtained directly from the back scattered fields.

\section{CONCLUSION}

Using the field equivalence principle, originally applied to non chiral media, the extended boundary condition method has been shown to be applicable to the evaluation of the scattered field and RCS of chirally coated 3D-scatterers. The formulation is versatile enough to handle complex shapes such as the fuselage of an aircraft.

\section{REFERENCES}

1.C.8.8onren, "Light scattering by an optically active spherell , Chem. Phys. Letters, 29, 3., 458-462 Dec, 1974.

2.A. Lakhtakia, V. K. Varadan and V. V. Varadan, "scattering and absorption characteristics of lossy dielectric chiral nonspherical objects.", Applied optics, Vol. 24, No. 23, PQ 4146-4154 Deo. 1985.

3.P.L.E. Uslenghi "scattering by an impedance sphere coated with a chiral layer", Electromagnetics 10:201-211, 1990 .

4.D.L.Jaggard and N. Engheta "Chirosorbth as an invisible medium**, Electronics Letters 25, 173-174,1989.

5. P. C. Haterman, "symmetry, unitarity and geometry in electromagnetic scattering, Phys. Rev. D. 3, 825, 1971.

6.P. Barber and C. Yeh, "soattering of electromagnetic waves by arbitrarily shaped dielectric bodies", Applied Optics $14,2864,1975$.

7.D. S. Wang and P. W. Barber. "Scattering by inhomogeneous nonspherical objects", Applied optics Vol.18, No. 8, po 11901197 April 1979.

8. J. A. Kong, Theory of electromagnetic wayes, Hiley, New York, 1975.

9.J. A. Stratton, Electromagnetic theory McGraw Kill, New York 1941 .

10.P. M. Morse and H. Eeshoach, Methods of theoretical physics Vo1.2 McGraw Hill New York 1953.

11. A. Lakhtakia, V.K. Varadan, V.V. Varadan, Time harmonic electromagnetic fields in chiral media, springer Verlag 1989. 